\title{
Review of: "Intact double stranded RNA is mobile and triggers RNAi against viral and fungal plant pathogens"
}

\author{
Francisco Murilo Zerbini ${ }^{1}$ \\ 1 Universidade Federal de Viçosa
}

Potential competing interests: The author(s) declared that no potential competing interests exist.

The manuscript by Brosnan et al. reports a series of experiments designed to assess the fate of topicallyapplied dsRNA in plants, and the efficiency of said dsRNA as an RNA interference (RNAi) trigger. RNAi is known to be induced by the presence of dsRNA in plant cells, either via expression from a transgene or as a result of viral infection. Therefore, it has been hypothesized that the topical application of dsRNA molecules would also trigger RNAi. This would be advantageous since RNAi would be induced in nongenetically modified plants, circumventing society's negative feelings about GM plants (although GM plants expressing an inverted-repeat transgene that would be transcribed as dsRNA are absolutely safe in every aspect - but this is another story). Previous work (properly cited in the manuscript) has shown that application of dsRNA in different plant organs will induce a localized RNAi response. However, RNAi needs to be systemic for the plant to be protected in the field. The experiments reported in this manuscript intended to determine whether dsRNA moves systemically once applied to a single leaf of the plant. The results indicate that dsRNA moves systemically through the apoplast, reaching root, reproductive and vegetative tissues. This was observed in different plant species, including model and crop plants. Also, RNAi was induced against fungi and viruses. Thus, the manuscript provides strong evidence for the effectiveness of the topical application of dsRNA in plants as a control strategy for pathogens.

I have no issues with the experimental design and with the author's interpretation of the results. Minor, specific (mostly editorial) comments are provided to improve the manuscript's presentation. My only issue is with the author's claim that the strategy is sustainable (P1L11, P2L11, P2L17). The kits used to synthesize dsRNA are expensive, and application may require the use of potentially toxic penetrants and other chemicals. Until a proper cost and environmental impact assessment is performed, I am not convinced that the application of dsRNA in the field is sustainable. I suggest that the text is modified to make this clear. Of course it may turn out to be sustainable (and I hope it does), but as far as this reviewer is aware, studies that demonstrate sustainability have not yet been carried out.

Specific comments 
P2L6: Correct spelling of ARGONAUTE

P2L19: Although several reports have alluded to the potential of...

P3L14: Meselect (uppercase M)

P8L9: Tomato would be a better host - TMV kills N. benthamiana plants in a matter of days by inducing systemic necrosis. I suggest "Although N. benthamiana is a proper host for both viruses, dsRNA..."

P8L16: Not sure what "3-four" means.

P8L20-25: This seems to defeat the purpose of the experiment. It is stated in P8L6-8 that the use of the dsGFP:GUS was intended to "allow for the detection of primary siRNAs derived exclusively from exogenous dsRNA application and not secondary amplified pools of siRNA derived from the virus itself". So, does the result imply that what is being detected is secondary siRNA derived from the virus ?

P9L22: ... despite their clear functionality and the ready detection of graft transmissible endogenous...

P12L8: Specify which penetrant was used, otherwise the experiment cannot be properly replicated

P13L1: Correct spelling of Meselect 